\title{
Desafios da população indígena ao acesso à saúde no Brasil: revisão integrativa de
}

\section{literatura}

\author{
Challenges of the indigenous population to access to health in Brazil: integrative literature review \\ Desafíos de la población indígena para acceder a la salud en Brasil: revisión bibliográfica
}

integradora

Recebido: 22/02/2021 | Revisado: 26/02/2021 | Aceito: 15/03/2021 | Publicado: 22/03/2021

Francinete Gonçalves de Oliveira ORCID: https://orcid.org/0000-0002-2853-9636 Singular Educacional, Brasil E-mail: francineteoliveira2008@yahoo.com.br

Paulo César Pereira de Oliveira ORCID: https://orcid.org/0000-0002-4600-5478 Singular Educacional, Brasil

E-mail: paulocezaroliveirafilho@gmail.com

Raimundo Nonato Barbosa de Oliveira Filho ORCID: https://orcid.org/0000-0003-1878-298X Singular Educacional, Brasil E-mail: rbfilho12@gmail.com

Heriederson Sávio Dias Moura ORCID: https://orcid.org/0000-0001-7654-2402 Universidade de São Paulo (USP-RP), Brasil E-mail: heriederson@gmail.com Dina Gaspar da Silva ORCID: https://orcid.org/0000-0003-1471-2191 Singular Educacional, Brasil E-mail: gaspardina41@gmail.com

Raphaela Cecília Cordeiro Lima ORCID: https://orcid.org/0000-0001-6004-5137 Universidade Paulista, Brasil E-mail: rafaelarocha148@gmail.com

Maria Leila Fabar dos Santos ORCID: https://orcid.org/0000-0003-1697-7599 Universidade Paulista, Brasil

E-mail: leila.fabar@hotmail.com

\begin{abstract}
Resumo
Este estudo teve como objetivo analisar os desafios enfrentados pela população indígena ao acesso à saúde. Trata-se de um estudo do tipo de revisão integrativa de literatura (RIL) pelo método de Ganong (1987), que propõe um processo de sistematização e análise dos dados, objetivando a compreensão do tema em estudo. Foram arrolados 11 artigos das bases de dados SciELO e LILACS, completos e com a temática sobre como se dá o enfrantamento da população indígena frente as dificuldades enfrentadas no acesso à saúde. Os indígenas tem enfrantado como principais dificuldades no tempo de permanência nas casas de apoio ou quando há necessidade de internação hospitalar; no rompimento da dieta, pois na aldeia baseia-se em alimentos provenientes da caça e pesca; dificuldades quanto a marcação de consulta e no deslocamento de onde vivem para a casa de apoio ao indígena; há dificuldades após o atendimento ou procedimentos necessários, pois acabam comprando os fármacos com os próprios recursos financeiros e nem sempre tem. Quanto a assistência da equipe de enfermagem, percebeu-se o despreparado para lidar com os costumes e questões culturais desses povos; contudo, muitos indígenas demonstraram satisfação no atendimento recebido. O presente estudo mostrou que os indígenas ainda enfrentam grandes desafios, principalmente quanto ao fator cultural, pois ainda sofrem com no acesso a assistência à saúde e na interação entre indígenas com profissionais não indígenas, devido a pela alta rotatividade. Apesar dessas observações, alguns estudos mostram que os profissionais facilitaram o acesso as casas de saúde e sua estadia no local de apoio.
\end{abstract}

Palavras-chave: Povos indígenas; Saúde indígena; Atenção primária à saúde.

\begin{abstract}
This study aimed to analyze the challenges faced by the indigenous population in accessing health care. This is a study of the type of integrative literature review (ILR) by the method of Ganong (1987), which proposes a process of systematization and analysis of data, aiming at the understanding of the subject under study. We enrolled 11 articles
\end{abstract}


from SciELO and LILACS databases, complete with the theme of how the indigenous population faces difficulties in access to health care. The main difficulties faced by the indigenous population are: the length of stay in the support homes or when there is a need for hospitalization; the disruption of their diet, because in the village it is based on food derived from hunting and fishing; difficulties in making appointments and in moving from where they live to the indigenous support home; there are difficulties after receiving care or necessary procedures, because they end up buying drugs with their own financial resources and do not always have them. As for the assistance given by the nursing team, we noticed that they were not prepared to deal with the customs and cultural issues of these people; however, many indigenous people showed satisfaction with the care they received. The present study showed that indigenous people still face great challenges, especially regarding the cultural factor, because they still suffer with the access to health care and the interaction between indigenous and non-indigenous professionals, due to the high turnover. Despite these observations, some studies show that professionals have facilitated the access to health care facilities and their stay at the support site.

Keywords: Indigenous peoples; Indigenous health; Primary health care.

\section{Resumen}

Este estudio tenía como objetivo analizar los retos a los que se enfrenta la población indígena para acceder a la atención sanitaria. Se trata de un estudio de revisión bibliográfica integradora (ILR) que utiliza el método de Ganong (1987), que propone un proceso de sistematización y análisis de datos, con el objetivo de comprender el tema estudiado. Se inscribieron 11 artículos de las bases de datos SciELO y LILACS, completos y con el tema de cómo la población indígena enfrenta las dificultades de acceso a la salud. Las principales dificultades que enfrentan los indígenas son el tiempo de permanencia en las casas de apoyo o cuando hay necesidad de hospitalización; la alteración de su dieta porque en el pueblo se basa en los alimentos derivados de la caza y la pesca; las dificultades para hacer citas y para trasladarse de donde viven a la casa de apoyo indígena; hay dificultades después de recibir atención o procedimientos necesarios porque terminan comprando los medicamentos con sus propios recursos económicos y no siempre los tienen. En cuanto a la asistencia prestada por el equipo de enfermería, se observó que no estaban preparados para tratar las costumbres y las cuestiones culturales de estos pueblos; sin embargo, muchos indígenas demostraron su satisfacción con la atención recibida. El presente estudio demostró que los indígenas todavía se enfrentan a grandes retos, especialmente en lo que respecta al factor cultural, ya que siguen sufriendo el acceso a la atención sanitaria y la interacción entre los profesionales indígenas y no indígenas debido a la alta rotación. A pesar de estas observaciones, algunos estudios demuestran que los profesionales facilitan el acceso a los centros sanitarios y su estancia en el lugar de apoyo.

Palabras clave: Pueblos indígenas; Salud indígena; Atención primaria de salud.

\section{Introdução}

De acordo com o último censo do Instituto Brasileiro de Geografia e Estatística (IBGE, 2010), há mais de 890 mil indígenas distribuídos em diferentes localidades no território brasileiro, correspondendo a 0,4\% da população brasileira. Essa população, distribuída em todos os estados brasileiros, está organizada em aproximadamente 505 terras indígenas, ocupando 12,5\% do território nacional (Mendes et al., 2018).

Brasil (2016), refere que os povos indígenas pensam e tem modos diferentes entre si, assim como daqueles que não são indígenas. Cada tribo possui cultura própria e formas de viver diferenciadas, por isso, o modo de entender a doença pode estar associada as suas crendices. Essa população em particular, entende que podem ser tratadas através do método terapêutico e de suas próprias crenças e nos conhecimentos que possuem. De acordo com Mendes et al. (2018), ainda que sejam caracterizados como minoria comparado ao total da população brasileira, os indígenas apresentam imensa sócio diversidade representada por 305 grupos étnicos falantes de 274 línguas.

Essa população compartilha entre si características sociais e históricas essenciais para entender sua vida hoje e "configuram como um dos segmentos mais desfavorecidos do ponto de vista econômico, habitacional, educacional e dos indicadores de saúde" (IBGE, 2010).

Em 1978, a Convenção de Alma Ata foi o marco nas discussões acerca da atenção primária à saúde (APS) como base para a efetivação da saúde enquanto direito fundamental e a redução das desigualdades estabelecidas entre povos. Durante os anos de 1980, diversos movimentos foram instituídos em busca de mudanças para o sistema de saúde brasileiro, dentre as ações foi instituído o Sistema Único de Saúde (SUS) (PAHO, 2007). 
No âmbito do SUS, a criação do subsistema de atenção à saúde indígena (SASI) e a Política de Atenção à Saúde dos Povos Indígenas (PNASPI) tornaram-se estratégias que garantem o acesso dos indígenas à saúde (Mendes et al., 2018), fatores que ressaltam a necessidade de que "a atenção à saúde se dê de forma diferenciada, levando-se em consideração as especificidades culturais, epidemiológicas e operacionais desses povos” (Fundação Nacional de Saúde, 2002).

A PNASPI estabelece a implantação de um modelo complementar e diferenciado de organização dos serviços voltados para a proteção, promoção e recuperação dá saúde (Pontes et al., 2015). Sua operacionalização se dá 8 através de 34 sistemas locais de saúde denominados Distritos Sanitários Especiais Indígenas (DSEI).

O DSEI consiste em um modelo de ordem de serviços que envolve um conjunto de atividades técnicas, visando medidas qualificadas e racionalizadas de assistência à saúde, promovendo a reordenação das práticas sanitárias, da rede de saúde e desenvolvendo atividades administrativo-gerenciais necessárias a prestação da assistência, com controle social (Brasil, 2016).

No Brasil, os povos indígenas vivem em lugares e em condições muito diferentes. Assim, os ambientes, o clima, o tipo de vegetação, a quantidade e os tipos de rios e fontes de água em cada região serão também diferentes. E cada povo tem a sua história, com experiências de contato e convívio com não índios também diversas (Brasil, 2016).

Diversas são as dificuldades que os povos indígenas enfrentam, dentre essas a situação fundiária não pode ser desprezada, pois esse fator influência nas condições de vida e saúde dessa população. Os povos indígenas e suas lideranças sabem que a regularização da terra é importante para uma boa condição de saúde, por isso as demandas pela regularização das terras indígenas aparecem com frequência nos relatórios finais das conferências nacionais de saúde indígena (Brasil, 2016).

À saúde indígena passou a ser garantida por meio da reforma da constituição em 1988, isto não se deve somente ao fato de que o atendimento à saúde do índio passou a fazer parte do SUS, que tem em vista o acesso igualitário aos serviços de saúde como também ganhou direitos ao acesso diferenciado a estes serviços como forma de admitir e garantir as formas organizacionais como costumes, língua nativa, crenças e tradições, assim como garantiram participação na proposição e deliberação a respeito das políticas de saúde por meio da criação de conselhos responsáveis (Novo, 2011).

Esta pesquisa teve como objetivo analisar através da literatura existente sobre os desafios enfrentados pela população indígena ao acesso à saúde, tendo como objetivos específicos (a) descrever as principais dificuldades encontradas por esta população; (b) investigar as dificuldades que os profissionais de saúde enfrentam durante a assistência prestada aos indígenas.

Esta abordagem se justifica como possibilidade de reflexão sobre a relação dos indígenas frente as dificuldades vivenciadas, de modo a oferecer informações pertinentes para possíveis tomadas de decisões pelos órgãos competentes, bem como futuras pesquisas de campo referente aos impactos sofridos por esta população nos aspectos físicos, bem como nas dimensões culturais e sociais.

\section{Metodologia}

Trata-se de uma revisão integrativa de literatura, cuja escolha se deu por oportunizar um embasamento científico que permiti através dos estudos já realizados identificar, analisar e sintetizar resultados pertinentes a área de saúde uma área específica de estudo. Este método facilita a identificação de resultados que possam contribuir para o direcionamento de futuras pesquisas e auxiliar o profissional a escolher condutas e a tomar decisões sobre determinado caso (Almeida, 2016).

De acordo com a proposta de Laurence Ganong (1987), a revisão integrativa está sistematizada em seis passos: (1) definição do tema/pergunta; (2) definição dos critérios de inclusão do estudo e seleção da amostra; (3) definição das informações a serem extraídas dos estudos em formato de tabelas, considerando todas as características em comum; (4) análise crítica dos resultados e avaliação dos estudos; (5) interpretação dos resultados; (6) apresentação das evidências encontradas. 
No intuito de analisar as publicações elencadas para esta pesquisa e identificar quais aspectos estão interligados a mesma, verifica-se que se trata de uma pesquisa com método qualitativo, pois a leitura das publicações permitirá que haja melhor interpretação e compreensão sobre a temática e sejam consideradas as opiniões relevantes acerca dos fenômenos em estudo pelo pesquisador (Pereira et al., 2018). Para dar seguimento as etapas descritas anteriormente, identificou-se e elaborouse a pergunta norteadora da referida pesquisa: Quais as dificuldades enfrentadas pela população indígena no acesso à saúde no Brasil?

Buscou-se publicações indexadas na Biblioteca Virtual em Saúde (BVS) em duas bases de dados: Literatura LatinoAmericana e do Caribe em Ciências da saúde (LILACS) e Scientific Electronic Library Online (SciELO). As buscas foram realizadas no mês de setembro de 2020 para a seleção e identificação de publicações. Para a busca de publicações nos periódicos utilizou-se os seguintes descritores: "Desafios população Indígena", "Saúde Indígena" e "Acesso à saúde". Foram elaborados três grupos de palavras-chaves, combinadas pelo método booleano $O R$ e AND.

A partir desse conjunto de palavras-chave e para a busca dos artigos que integrariam esta revisão, através dos filtros das próprias bases de dados (Figura 1), estabeleceu-se os seguintes critérios de inclusão: ano de publicação (2014 a 2020), artigos completos, gratuitos e no idioma da língua portuguesa. Foram excluídos os artigos incompletos e duplicados. As referências foram digitadas e organizadas em planilha Excel para o controle dos critérios elencados.

A primeira etapa da avaliação das referências incluídas constou da leitura dos títulos dos artigos, a segunda da leitura dos resumos, por fim, a terceira etapa se deu pela leitura e avaliação na íntegra das publicações selecionadas. Eventuais desacordos foram discutidos a fim de se estabelecer critérios e entrar em comum acordo com temática.

Na primeira e segunda etapas foram excluídos os estudos cuja população alvo não era os indígenas, estudos empíricos e aqueles cujo acesso não estava disponível gratuitamente. Na terceira etapa, os artigos foram lidos na íntegra e, para se verificar a pertinência de sua inclusão, utilizou-se as informações: local de moradia, total da amostra, país da pesquisa, instrumentos utilizados para medida do desfecho, ocorrência de dificuldades ao acesso à saúde e educação, atuação do enfermeiro frente a atenção básica primária aos cuidados da saúde indígena e outras variáveis independentes e desfecho.

Figura 1. Fluxograma dos artigos selecionados após aplicação dos filtros nas bases de dados.

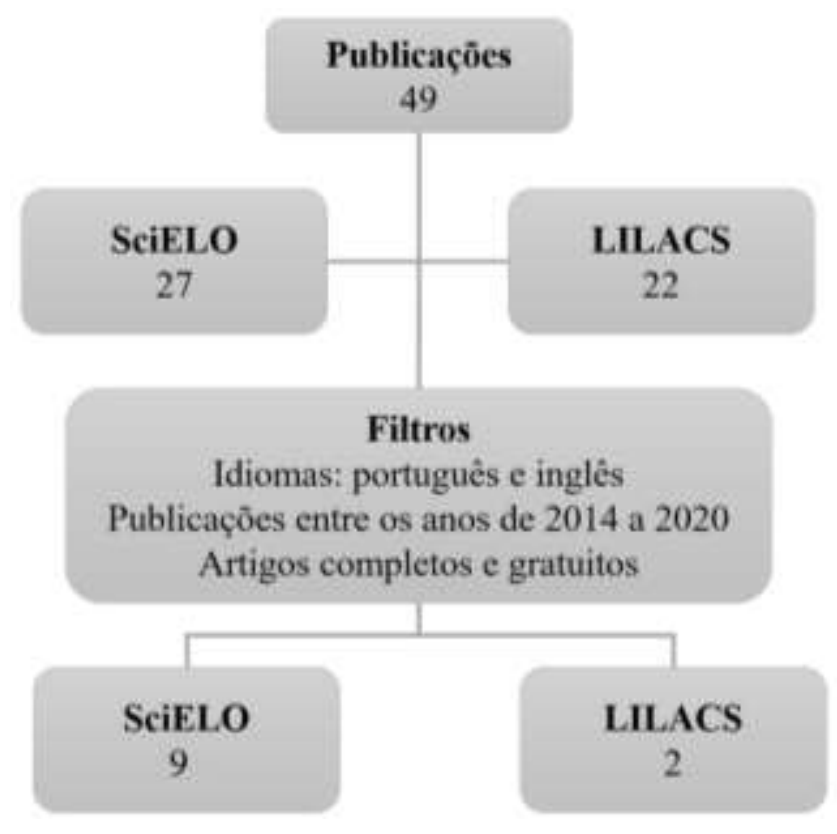

Fonte: Oliveira et al. (2021). 
O Quadro 1 mostra a distribuição dos artigos selecionados após aplicação dos critérios de inclusão e exclusão, optouse pela organização dos mesmos em ano, periódico, autor(es), título e desenho da pesquisa. O desenho das publicações selecionadas demonstra principalmente abordagens diretas ao público estudado, seja através de entrevistas semiestruturas, exame físico e bioquímico, como também análise secundária e pressupostos etnográficos (Quadro 1).

Quadro 1. Seleção de acordo com o ano, periódico, autor(es), título e desenho da pesquisa.

\begin{tabular}{|c|c|c|c|c|}
\hline Ano & Periódico & $\operatorname{Autor}(e s)$ & Título & Desenho da pesquisa \\
\hline 2014 & $\begin{array}{c}\text { Caderno de Saúde } \\
\text { Coletiva }\end{array}$ & Azevedo et al. & $\begin{array}{l}\text { O poder de acessar à saúde: uma } \\
\text { análise do acesso à saúde na etnia } \\
\text { indígena Xukuru do Ororubá, } \\
\text { Pesqueira (PE) }\end{array}$ & $\begin{array}{l}\text { Estudo de caso, utilizando-se } \\
\text { de entrevistas, grupos focais, } \\
\text { análise documental e } \\
\text { observação direta. }\end{array}$ \\
\hline 2015 & $\begin{array}{c}\text { Ciência \& Saúde } \\
\text { Coletiva }\end{array}$ & Pontes et al. & $\begin{array}{l}\text { O modelo de atenção diferenciada } \\
\text { nos Distritos Sanitários Especiais } \\
\text { Indígenas: reflexões a partir do } \\
\text { Alto Rio Negro/AM, Brasil. }\end{array}$ & $\begin{array}{l}\text { Pesquisa qualitativa, com } \\
\text { método de observação } \\
\text { participante, entrevistas e } \\
\text { análise documental. }\end{array}$ \\
\hline 2015 & $\begin{array}{c}\text { Rev Esc Enferm } \\
\text { USP }\end{array}$ & Borghi et al. & $\begin{array}{l}\text { Singularidades culturais: o acesso } \\
\text { do idoso indígena aos serviços } \\
\text { públicos de saúde* }\end{array}$ & 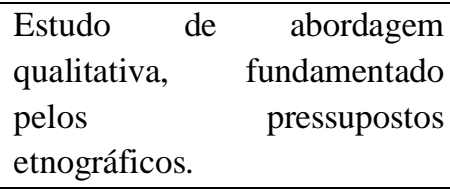 \\
\hline 2015 & $\begin{array}{l}\text { Cad. Saúde } \\
\text { Pública }\end{array}$ & Scopel et al. & $\begin{array}{l}\text { Intermedicalidade } \\
\text { protagonismo: a atuação dos } \\
\text { agentes indígenas de saúde } \\
\text { Munduruku da Terra Indígena } \\
\text { Kwatá-Laranjal, } \\
\text { Brasil Amazonas, }\end{array}$ & $\begin{array}{l}\text { Estudo de observação } \\
\text { participante e entrevistas } \\
\text { realizadas de } 2009 \text { a } 2011 . \\
\text { Entrevistas semiestruturadas }\end{array}$ \\
\hline 2015 & $\begin{array}{l}\text { Cad. Saúde } \\
\text { Pública }\end{array}$ & Fávaro et al. & $\begin{array}{l}\text { Obesidade e excesso de peso em } \\
\text { adultos indígenas Xukuru do } \\
\text { Ororubá, Pernambuco, Brasil: } \\
\text { magnitude, } \\
\text { socioeconômicos e demográficos } \\
\text { associados. }\end{array}$ & $\begin{array}{l}\text { Estudo de corte transversal e } \\
\text { base populacional através da } \\
\text { pesquisa de campo. }\end{array}$ \\
\hline 2016 & $\begin{array}{c}\text { Epidemiol. } \\
\text { Serviço e Saúde }\end{array}$ & Santos et al. & $\begin{array}{l}\text { Assistência prestada pelo Sistema } \\
\text { Único de Saúde de Teresina à } \\
\text { população indígena do Maranhão, } \\
\text { 2011: um estudo descritivo }\end{array}$ & 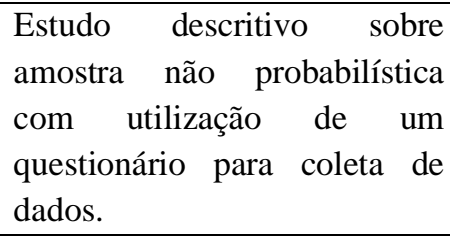 \\
\hline 2016 & $\begin{array}{c}\text { Saúde Social \& } \\
\text { Saúde }\end{array}$ & Silva et al. & $\begin{array}{l}\text { Dificuldades enfrentadas pelos } \\
\text { indígenas durante a permanência } \\
\text { em uma Casa de Saúde Indígena } \\
\text { na região Amazônica/Brasil }\end{array}$ & $\begin{array}{lr}\text { Estudo } & \text { qualitativo } \\
\text { exploratório, com abordagem } \\
\text { por meio de entrevistas } \\
\text { semiestruturadas, gravadas e } \\
\text { transcrita. }\end{array}$ \\
\hline 2018 & $\begin{array}{c}\text { Revista de Saúde } \\
\text { Publica }\end{array}$ & Graeff et al. & $\begin{array}{l}\text { Aspectos epidemiológicos da } \\
\text { infecção pelo HIV e da AIDS } \\
\text { entre povos indígenas }\end{array}$ & $\begin{array}{l}\text { Estudo descritivo. Os dados } \\
\text { foram extraídos do } 1 \text {. registros } \\
\text { provenientes dos atendimentos } \\
\text { diários das EMSI; } 2 \text {. banco de } \\
\text { dados do Sinan; 3. Sistema de } \\
\text { Informação de Atenção à } \\
\text { Saúde Indígena (SIASI). }\end{array}$ \\
\hline 2019 & Epidemiol. Serv. & Malacarne et al. & Acesso aos serviços de saúde para & Estudo transversal, mediante \\
\hline
\end{tabular}




\begin{tabular}{|c|c|c|c|c|}
\hline & Saúde & & $\begin{array}{l}\text { o diagnóstico e tratamento da } \\
\text { tuberculose entre povos indígenas } \\
\text { do estado de Rondônia, Amazônia } \\
\text { Brasileira, entre } 2009 \text { e 2011: um } \\
\text { estudo transversal* }\end{array}$ & $\begin{array}{l}\text { entrevistas, as dimensões } \\
\text { geográfica, econômica e } \\
\text { funcional do acesso aos } \\
\text { serviços de TB, apresentadas } \\
\text { descritivamente. }\end{array}$ \\
\hline 2020 & $\begin{array}{l}\text { Cad. Saúde } \\
\text { Pública }\end{array}$ & Chagas et al. & $\begin{array}{l}\text { Prevalência estimada e fatores } \\
\text { associados à hipertensão arterial } \\
\text { em indígenas adultos Krenak do } \\
\text { Estado de Minas Gerais, Brasil. }\end{array}$ & $\begin{array}{l}\text { Estudo transversal, com } \\
\text { abordagem de exame físico } \\
\text { (antropometria e aferição da } \\
\text { pressão arterial), bioquímico } \\
\text { (glicemia capilar) e de } \\
\text { questionários (variáveis } \\
\text { sociodemográficas, de estilo } \\
\text { de vida e hábitos alimentares). }\end{array}$ \\
\hline 2019 & $\begin{array}{l}\text { Ciênc. saúde } \\
\text { coletiva }\end{array}$ & $\begin{array}{l}\text { Nascimento et } \\
\text { al. }\end{array}$ & $\begin{array}{l}\text { Desafios na formação de } \\
\text { enfermeiros indígenas em Mato } \\
\text { Grosso, Brasil }\end{array}$ & $\begin{array}{l}\text { Estudo exploratório } \\
\text { qualitativo }\end{array}$ \\
\hline
\end{tabular}

Fonte: Oliveira et al. (2021).

\section{Resultados e Discussão}

\subsection{Principais dificuldades enfrentadas pelos povos indígenas frente ao acesso à saúde}

O estudo de Pontes et al. (2015), refere que uma das principais dificuldades que os indígenas têm enfrentado está inerente ao tempo de permanência na casa de apoio ou quando há necessidade de internação hospitalar, por haver rompimento da dieta que eles têm na aldeia que se baseia em alimentos da caça e pesca. Ele também descreve a diferença entre os hábitos alimentares daqueles considerados não indígenas aos dos indígenas, causando-lhes dificuldades na questão alimentar.

$\mathrm{O}$ estudo supracitado descreve ainda alguns fatores que causam tristeza nos indígenas, sendo esses o sentimento de deixar o convívio familiar diário, afastamento em detrimento da necessidade por alguns períodos de tempo e preocupação com os familiares. Dessa forma, esses fatores têm impacto direto na recuperação da saúde daqueles que necessitam, pois a família é tida como elo primordial se tratando da condição de saúde desses indivíduos.

Estudo semelhante realizado por Silva et al. (2016), reporta que a procura pelos serviços de média e alta complexidade tem como principais motivos a necessidade da realização de retorno para exames especializados, acompanhamento médico e alguns procedimentos hospitalares. Através da entrevista foi possível constatar que as maiores dificuldades enfrentadas pelos indígenas foram: dificuldade financeira, seja para realizar consultas particulares ou adquirir medicamentos, marcação de consultas, demora para procedimentos hospitalares, barreira linguística e cultural, dificuldade para acesso a alimentação tradicional da sua culinária, dentre outros fatores.

Diferentemente da pesquisa realizada acima, Azevedo et al. (2014) relata que as dificuldades que o povo Xukuru enfrenta está além do acesso à saúde, como os conflitos locais e sociais que transcendem as barreiras culturais, interferindo na luta pela terra ocupada. Pode se evidenciar que os capitais ativos no campo da saúde indígena (social, cultural, étnico, político e simbólico), especificamente na atenção básica, constituíram recursos de poder associados ao acesso.

Por sua vez, Borghi et al. (2015), descreve as dificuldades de acesso dos indígenas idosos que buscam por acesso à saúde junto ao SUS. O estudo de Silva et al. (2016) mostra resultados positivos opostos, relata que mesmo com as dificuldades existentes, os idosos que saíram em busca de assistência médica nas unidades básicas de saúde encontraram facilidade, seja nas consultas médicas, odontológicas, consultas de enfermagem ou quaisquer outros procedimentos. Afirma ainda que a equipe de 
enfermagem participa de modo eficaz no processo de assistência ao idoso, estando presente em ações que antecedem as consultas médicas, prestando cuidados e encaminhando-os para o serviço de referência quando necessário.

Porém, a distância de algumas residências à UBS e o horário de funcionamento representou uma barreira para o acesso. $\mathrm{O}$ acesso aos serviços da rede de referência do SUS foi marcado pela demora no atendimento e encaminhamento a outros serviços especializados. A análise realizada demonstrou existência de falhas nos serviços de saúde para o atendimento diferenciado, como a ausência de cuidados congruentes às necessidades culturais do idoso indígena e a dificuldade de comunicação entre profissional/paciente, em razão do idioma Kaingang (Borghi et al., 2015).

Tais evidencias denotam a importância de se conhecer as especificidades culturais de cada etnia, e assim poder oferecer oportunidades de acesso à saúde, como também o cuidado integral que respeite as diferenças culturais de cada povo.

A respeito do acolhimento na Casa de Apoio ao povo indígena - Casai/Piaú, os dados apresentados receberam indígenas em busca de atendimento de média a alta complexidade. Em estudo realizado por Santos et al. (2016), os participantes comparam os atendimentos prestados no estado do Piauí, relatam a grande diferença na assistência pela Casai ao SUS. Uma vez que, na Casai eles conseguem manter os costumes tradicionais das aldeias, especialmente, no que se refere a alimentação e hábitos de dormir, apesar disso consideram o atendimento no SUS como "boa e muito boa", apontando como maior dificuldade a distância para chegar aos locais de serviços disponibilizados pelo Casai.

Na pesquisa realizada por Silva et al. (2016), mostra que na Casai/Santarém as principais dificuldades encontradas estão relacionadas a privacidade de cada etnia e estrutura física. Dessa forma, sugerem no estudo que seja ofertado aos indígenas quartos separados, agilidade nas consultas médicas, encaminhamento para as unidades de referência, apoio financeiro para adequar os hábitos alimentares e realizarem a compra de medicações.

Corroborando com o estudo supracitado, Malacarne et al. (2019), mostra que os indígenas da região de Cacoal e Guajará-Mirim diagnosticados com tuberculose e outras doenças, têm como principal queixa a falta de dinheiro para o deslocamento, falta de transporte para apoio ao indígena, assim como a dimensão geográfica, pois o território onde habitam é localizado um pouco longe da Casai/Porto Velho. Os indígenas relatam ainda sentir ausência de profissionais da saúde para atendimento onde moram, sendo está uma barreira relevante para o encaminhamento as unidades de referência à saúde.

Referente aos quadros de saúde dos indígenas em diferentes regiões do Brasil, na pesquisa realizada por Graeff et al. (2018), os Guarani e Kaiowá, com o território indígena localizado no Mato Grosso do Sul, apresentam um quadro de saúde bastante complexo, com alta prevalência de doenças infecciosas, elevada mortalidade infantil e desnutrição, juntamente com a prevalência crescente de doenças crônicas não transmissíveis.

O estudo supracitado mostra ainda que as dificuldades encontradas estão relacionadas, especialmente, a questão da mobilidade geográfica, eles estão localizados em uma faixa de fronteira, uma vez que o território Guarani e Kaiowá se estendem até o Paraguai, fator que prejudica o acesso regular aos serviços de saúde, constituindo desta forma fatores de risco para a circulação de diversas doenças transmissíveis. Além disso, o autor descreve que as situações de intenso contato interétnico e a marginalização no acesso aos serviços de saúde, constituem fatores de risco para a circulação e propagação de doenças como HIV entre os povos indígenas.

Fávaro et al. (2015), mostra em sua pesquisa que o povo indígena Xukuru do Ororubá apresenta elevada prevalência de sobrepeso $(33,5 \%)$ e obesidade $(14,8 \%)$ em homens e mulheres adultos. Tais resultados são semelhantes aos encontrados por Graeff et al. (2018), no qual apresentou frequências próximas entre homens (22\%) e mulheres (40\%).

A pesquisa de Graeff et al. (2018), mostra ainda que 58,2\% dos homens e 66,9\% das mulheres das etnias Teréna, Guarani e Kaiowá apresentaram sobrepeso, por sua vez, a taxa de obesidade em mulheres foi praticamente o dobro (30,8\%) quando comparado aos homens $(14,2 \%)$. 
As pesquisas citadas acima, mostraram que os determinantes do agravo nutricional em indígenas estão intimamente ligados as transformações ocorridas por fatores demográficos, culturais, econômicos, ambientais, assim como pela decorrência de patologias que foram ignoradas na assistência à saúde e políticas públicas.

Grande parte das doenças são acometidas pelos indígenas devido à falta de cuidados médicos, a busca tardia por assistência à saúde, assim como pela prevalência de doenças transmissíveis e hipertensão arterial. De acordo como Chagas et al. (2020), os fatores associados as doenças encontradas nos indígenas Krenak são semelhantes à de outros povos indígenas brasileiros, podendo estar associada as mudanças que ocorreram no estilo de vida, acesso a alimentos industrializados, mudanças sociodemográficas, bem como pelas situações de adversidades na vida desses povos nas últimas décadas.

\subsection{Ações dos profissionais de saúde no território indígena}

No estudo de Santos et al. (2016), as maiores dificuldades encontradas estão relacionadas a dificuldades da compreensão da língua e seus dialetos, resistência dos indígenas em participar do estudo, o que dificultou atingir o públicoalvo na sua totalidade, entretanto, as dificuldades foram atenuadas pela intervenção dos profissionais da Casai, auxiliando os indígenas a compreender a importância da pesquisa e facilitando o diálogo entre pesquisadores e indígenas.

Em estudo de Scopel et al. (2015), realizado no município de Borba/Amazonas, os agentes indígenas tinham papel central na promoção da atenção primária ao desempenharem atividades que incluíam remoção e acompanhamento de pacientes, visitas domiciliares, programas de controle de doenças crônicas e acompanhamento nutricional das crianças, bem como indicação de especialistas indígenas e "remédios caseiros".

Scopel et al. (2015), relata ainda que os agentes indígenas atuavam na capacitação daqueles recém-contratados e tiveram participação ativa em reuniões do Conselho Local e Distrital de Saúde, nas negociações junto aos políticos locais para reivindicar recursos financeiros, ou em reuniões com órgãos de diferentes esferas da administração pública.

A inserção de agentes indígenas nas equipes de saúde garantiria uma adequação cultural. Para Pontes et al. (2015), os principais desafios encontrados neste estudo estão relacionados as especificidades culturais, mesmo com diversas críticas, torna-se fundamental a inserção e ampliação dos profissionais indígenas no sistema, pois eles reconhecem as práticas e têm conhecimento sobre o território.

Observa-se também que o acesso à saúde do idoso indígena constitui um desafio na construção do cuidado integral desse indivíduo, além de ser um elemento importante para a gestão e avaliação de serviços de saúde indígena. Assim, o enfermeiro tem entre suas atribuições promover o acesso por esse grupo, esse profissional assume papel primordial para estabelecer estratégias em saúde e proporcionar ampliação do acesso aos serviços ofertados (Borghi et al., 2015).

Para Nascimento et al. (2019), a equipe de enfermagem desenvolve junto à essa população um trabalho que se baseia no respeito à cultura e tradições, fortalecendo os vínculos entre profissionais de saúde e lideranças da comunidade indígena.

\section{Considerações Finais}

Os achados desta pesquisa permitiram identificar que a população indígena ainda enfrenta grandes desafios relacionados ao fator cultural, uma vez que determinados locais os seus costumes e crenças não são respeitadas e, por vez, ignoradas durante a prestação de cuidados.

Além disso, os serviços de saúde se encontram geograficamente distantes do local de moradia desses povos, fator que corrobora para o difícil acesso aos atendimentos necessários. Observou-se que a alta rotatividade de profissionais interfere na interação com os indígenas, embora alguns estudos tenham mostrado o acesso as CASAI's são facilitadas ao pessoal de saúde, sendo ofertada ainda a estadia nestes locais. 
A prevalência de indígenas acometidos por diferentes tipos de doenças se mostrou elevada, podendo ser citado as infecções sexualmente transmissíveis, hipertensão arterial, assim como doenças oriundas da mudança do estilo de vida e sociodemográficas.

A incompreensão e conflitos de dimensão cultural, ausência do entendimento da linguística dentre outros fatores culturais são dificuldades encontradas por profissionais de para a atuação no território indígena. Porém, há controvérsia relacionada a este motivo, ora sendo satisfatório, ora não.

Contemplando as particularidades da temática, os resultados apontam para a realização de estudos posteriores nos diversos âmbitos de assistência à saúde, considerando uma diversidade de territórios indígenas, pois os estudos nesta área são limitados. Assim, os profissionais de saúde poderão ter subsídios teórico para melhor atender populações indígenas, de forma a prestar um serviço holístico e com equidade.

\section{Referências}

Azevedo, A. L. M., Gurgel, I. G. D., \& Tavares, M. A. (2014). O poder de acessar à saúde: uma análise do acesso à saúde na etnia indígena Xukuru do Ororubá, Pesqueira (PE). Cadernos Saúde Coletiva, 22(3), 275-280.

Borghi, A. C., Alvarez, A. M., Marcon, S. S., \& Carreira, L. (2015). Singularidades culturais: o acesso do idoso indígena aos serviços públicos de saúde. Rev Esc Enferm USP, 49(4): 589-595.

Brasil. Fundação Nacional de Saúde. (2002). Política Nacional de Atenção à Saúde dos Povos Indígenas. Ministério da saúde: Fundação Nacional de Saúde.

Brasil. Ministério da saúde. (2016). Secretaria de Gestão do Trabalho e da Educação nà saúde. Departamento de Gestão da Educação nà saúde. Programa de Qualificação de Agentes Indígenas de Saúde (AIS) e Agentes Indígenas de Saneamento (AISAN) / Ministério da saúde, Secretaria de Gestão do Trabalho e da Educação nà saúde, Departamento de Gestão da Educação nà saúde. - Brasília: Ministério da saúde.

Chagas, C. A., Castro, T. G., Leite, M. S., Viana, M. A. C. B. M., Beinner, M. A., \& Pimenta, A. M. (2020). Prevalência estimada e fatores associados à hipertensão arterial em indígenas adultos Krenak do Estado de Minas Gerais, Brasil. Cad. Saúde Pública, $36(1)$ : e00206818.

Fávaro, T. R., Santos, R. V., Cunha, G. M., Leite, I. C., \& Coimbra Jr., C. E. A. (2015). Obesidade e excesso de peso em adultos indígenas Xukuru do Ororubá, Pernambuco, Brasil: magnitude, fatores socioeconômicos e demográficos associados. Cad. Saúde 20 Pública, 31(8): 1685-1697.

Ganong, L.H. (1987). Integrative reviews of nursing research. Research in Nursing \& Health, 10(11): 1-11.

Graeff, s. V. B., Pícolli, R. P., Arantes, R., Castro, V. O. L., \& Cunha, R. V. (2019). Aspectos epidemiológicos da infecção pelo HIV e da aids entre povos indígenas. Revistà saúde Pública, 53: 71.

IBGE. Instituto Brasileiro de Geografia E Estatística. (2010). Os indígenas no censo demográfico 2010: primeiras considerações com base no quesito cor ou raça. Brasília: IBGE. http://www. ibge.gov.br/indigenas/indigena_censo2010.pdf.

Malacarne, j., Gava, C., Escobar, A. L., Souza-Santos, R., \& Basta, P. C. (2019). Acesso aos serviços de saúde para o diagnóstico e tratamento da tuberculose entre povos indígenas do estado de Rondônia, Amazônia Brasileira, entre 2009 e 2011: um estudo transversal. Epidemiol. Serv. Saúde, $28(3)$ : e2018231.

Mendes, A. M., Leite, M. S., Langdon, E. J., \& Grisotti, M. (2018). O desafio da atenção primária nà saúde indígena no Brasil. Revista Panamericana Salud Publica, 42: e184.

Nascimento, V. F., Hattori, T. Y., \& Trettel, A. C. P. T. (2019). Desafios na formação de enfermeiros indígenas em Mato Grosso, Brasil. Ciência \& Saúde Coletiva, 25(1): 47-56.

Pereira, A. S., Shitsuka, D. M., Parreira, F. J. \& Shitsuka, R. (2018). Metodologia da pesquisa científica. UFSM. https://repositorio.ufsm.br/bitstream/handle/1/15824/Lic_Computacao_Metodologia-Pesquisa-Cientifica.pdf?sequence=1.

Pontes, A. L. M., Garnelo, S. R., \& Garnelo, L. (2015). O modelo de atenção diferenciada nos Distritos Sanitários Especiais Indígenas: reflexões a partir do Alto Rio Negro/AM, Brasil. Ciência \& Saúde Coletiva, 20(10): 3199-3210.

Santos, M. M., Cruz, K. J. C., Sá, L. C. R., Batista, C. C., Aguiar, E. M. G., \& Nogueira, A. M. T. (2016). Assistência prestada pelo Sistema Único de Saúde de Teresina à população indígena do Maranhão, 2011: um estudo descritivo*. Epidemiol. Serv. Saúde, 25(1): 127-136.

Scopel, D., Scopel, R. P. D., \& Langdon, E. J. (2015). Intermedicalidade e protagonismo: a atuação dos agentes indígenas de saúde Munduruku da Terra Indígena KwatáLaranjal, Amazonas, Brasil. Cad. Saúde Pública, 31(12): 2559-2568.

Silva, d. M., Nascimento, E. H. S., Santos, L. A., Martins, N. V. N., Sousa, M. T., \& Figueira, M. C. S. (2016). Dificuldades enfrentadas pelos indígenas durante a permanência em uma Casa de Saúde Indígena na região Amazônica/Brasil. Saúde Soc., 25(4): 920-929. 